\title{
Psychological Impacts Among Yemeni Healthcare Workers During the COVID-19 Pandemic: an Electronic-based, Cross-sectional Analytical Survey
}

\section{Gamil Ghaleb Alrubaiee}

Al-Razi University

Farouk Abdulrahman Al-Qadasi

Health Officer, International Organization of Migration

Talal Ali Hussein Al-Qalah

Al-Razi University

Jennifer Ann Cole

Royal Holloway University of London

Yaser abdullah Ghaleb Alburiahy

Modern European Hospital

Mohammed Alsabri ( $\nabla$ alsabri5000@gmail.com )

Al-Thawra Modern General Teaching Hospital

\section{Research Article}

Keywords: SARS-CoV-2, COVID-19, stress, anxiety, insomnia, depression, well-being, health care workers, Yemen

Posted Date: September 14th, 2021

DOl: https://doi.org/10.21203/rs.3.rs-789394/v1

License: (9) This work is licensed under a Creative Commons Attribution 4.0 International License. Read Full License 


\section{Abstract}

Background: Yemeni HCWs face higher levels of psychological stress than others in the fight against the COVID-19 pandemic due to the country's war and internal conflict. The current study investigates the levels of stress, anxiety, insomnia, depression, and well-being among Yemeni HCWs during the COVID-19 pandemic.

Methods: A web-based, cross-sectional survey was used among 1,248 Yemeni HCWs. An electronic selfreported questionnaire was used to collect data. The URL link was distributed to the HCWs via social media and emails. The Statistical Package for Social Sciences (IBM SPSS), version 24.0 was used in managing and analyzing the data collected. T-test and one-way ANOVA were used to compare the participants' data and independent variables. Spearman's correlation coefficients were used to determine the relationship between study variables. A p-value of $<0.05$ with a $95 \%$ confidence interval was considered statistically significant. The survey was conducted between 6 November 2020, 11 PM to 3 April 2021, 11 PM.

Results: The results indicated that 73.0, 57.3, 49.8, 53.2, and $85.2 \%$ of all HCWs reported symptoms of moderate or severe stress, insomnia, anxiety, depression, and worse quality of life, respectively. Out of ten independent variables, five were statistically significant (years of experience, working hours per day, marital status, age, and working days per week) $(p<0.05)$. The results further showed significant positive correlations between stress and scores of anxiety, insomnia, and depression and also between anxiety and insomnia and depression and between insomnia and depression. There was significant negative correlation between wellbeing scores and scores of stress, anxiety, insomnia, and depression.

Conclusion: Yemeni HCWs experience considerable levels of stress, anxiety, depression, insomnia, and poor quality of life. Psychological support to build resilience to the prevailing conditions may need to be embedded into medical school training and continuing professional development to help retain HCWs within Yemen, as amongst the respondents where was a high percentage of HCWs under 40 years of age (85.8\%) and with less than 10 years' experience (72.7\%), a consequence of many more experienced medics leaving Yemen for safer and more secure jobs elsewhere.

\section{Introduction}

International Committee on Taxonomy of Viruses (ICTV) named a novel coronavirus SARS-CoV-2 that was first detected in Wuhan, Hubei Province, China, in December 2019 and is now known as COVID-19 [1]. As of March 11, 2020, the World Health Organization (WHO) declared COVID-19 a global pandemic [2]. The consequences of infection range from asymptomatic carriage to mild upper respiratory tract symptoms to a respiratory failure and multi-organ failure and can result in death [3], particularly in elderly patients and those with certain underlying health conditions, such as chronic lung or kidney disease. By 23rd July 2021, the virus had infected over 192 million people worldwide and the number of deaths had totaled more than 4 million people [4]. Close to 10 percent of all COVID-19 cases worldwide, according to 
the WHO, were linked to healthcare workers (HCWs) [5]. Furthermore, according to updated figures from the WHO, at least $115,000 \mathrm{HCWs}$ have died from COVID-19 since the beginning of the pandemic to 27 MAY, 2021[6].

Frontline HCWs play a vital role in the care of cases with COVID-19, making them more susceptible to infection and more likely to spread an infection to others without the use of infection control measures and personal protective equipment (PPE) [7]. Additionally, they have had to deal with increased patient numbers and overstretched healthcare systems during the pandemic. Stress is the most common environmental risk factor for psychiatric illnesses, and people who are exposed to it for an extended period of time are more likely to develop depression or other mental illnesses, which, among other negative health consequences, compromises their immune response, increasing the likelihood of infection and leading to more severe symptoms or even death [8]. One recent research paper reported that COVID-19 disease has been known to cause considerable mental health problems in infected patients, HCWs, families, children, and students [9]. Another study, which was conducted to assess the psychological impact of quarantine and how to reduce it, discovered that the increasing incidence of COVID-19 causes fear, anxiety, and panic among the community, particularly among HCWs, who are one of the groups most at risk for getting this novel SARS-CoV-2 infection [10]. Chen et al., (2021) and Li, Scherer, Felix, and Kuper (2021) also found that HCWs can suffer moderate to severe anxiety and depression at the same time. Consequently, the mental health of HCWs should be given more attention during the COVID-19 pandemic $[11,12]$.

Operating under conditions of psychological stress is commonplace for medics in Yemen. Over the past six years, ongoing war and civil unrest have severely impacted the country's infrastructure, including its health facilities. At the moment, Yemen's healthcare system is in a state of near collapse. According to WHO, only about half of Yemen's 5,056 pre-war health facilities are still operational, and even in those that are, there is a severe shortage of medical personnel and essential emergency medical equipment $[13,14]$. Despite the fact that the COVID-19 pandemic has exacerbated an already difficult situation, it is important to understand the impact of psychological stress on Yemen's healthcare workers so that appropriate interventions can be put in place to support psychological resilience in challenging situations. Recently, there have been calls for more research on the impacts of Yemeni HCWs' mental health on their ability to function [15] and on the humanitarian aid sector to provide more support for HCWs' mental health needs [16].

The struggle against COVID-19 begun for Yemeni medics on April 10th, 2020. Ash Shihr, Hadramout Province in southern Yemen, was the site of the first confirmed case. On April 29, 2020, five more cases of COVID-19 were confirmed and registered in Aden, Yemen's temporary capital (Sana'a is the traditional capital). Afterwards, cases in other cities began to increase on a daily basis. There were 4,247 confirmed cases as of April 7, 2021, with 882 deaths [17]. Yemen, with a critical shortage of healthcare professionals, and healthcare supplies, including basic PPE, and limited testing capacity, have the highest case fatality rate (29\%) in the world [18]. However, this figure is almost certainly underestimating because of the political situation and the country's fragile and weak health system. 
In the context of the COVID-19 response, HCWs in Yemen are exposed to a variety of occupational hazards that put them at risk of disease, injury, psychological disturbances, and even death, including (a) occupational infection with COVID-19; (b) skin disorders and heat stress from prolonged use of PPE; (c) exposure to toxic substances due to increased use of disinfectants; (d) psychological distress [19, 20]. In addition, the emergence of new strains of SARS-CoV-2, and the possibility of being these types transmitted to a highly devastating country, has triggered a huge further concern even among HCWs. To quantify the problem's scope, Yemen's first-line health care workers must be assessed for their mental health. This will highlight the need for psychological interventions to help anticipate and avoid the consequences that may arise during the epidemic's lifetime.

\section{Methods}

\section{Study design, area, and time period}

In a web-based cross-sectional survey, Yemeni healthcare workers who provide patient healthcare services were assessed about their levels of stress, anxiety, insomnia, depression, and overall well-being. The survey took place between November 6, 2020, at 11 p.m., and April 3, 2021, at 11 p.m.

\section{Study instrument}

A questionnaire adapted from previously published studies $[9,21]$ was used in the current study. The questionnaire comprises six sections; Section (1) is related to socio-demographic characteristics such as age, gender, marital status, living status, occupation, educational level, the current hospital worked in, and working unit in that hospital. Section (2) deals with respondent stress (10-items). Section (3) deals with respondent anxiety (8-items). Section (4) deals with respondent insomnia (7-items). Section (5) deals with respondent depression (9-items) and Section (6) deals with respondent well-being (5-items).

\section{Scoring of stress, anxiety, insomnia, depression, and well-being}

The stress level was assessed using a 5-point Likert scale. The scores were calculated by assigning scores of $0,1,2,3$ and 4, to the response levels 'never', 'almost never, 'sometimes', 'fairly often, and 'very often', respectively. For questions $4,5,7$, and 8 the scores were reversed $(0=4,1=3,2=2,3=1,4=0)$. Then, the scores were summed together for the ten questions. The total scores recorded ranged between 0 and 40 , with higher scores indicating higher levels. A score of $<14$ was considered low perceived stress, scores of 14-26 were considered moderate perceived stress, while a score of $>26$ was considered high perceived stress. Likewise, a 5-point Likert scale was used to rate insomnia items (e.g., $0=$ no problem; 4 $=$ very severe problem). Scores ranged between 0 and 28 . A score of $<8$ was considered no clinically significant insomnia, 8-14 was considered mild insomnia, 15-21 was considered moderate insomnia, while a score of $\geq 22$ was considered severe insomnia. Anxiety levels were assessed using a 4-point Likert scale, with the severity of anxiety calculated by allocating scores of $0,1,2$, and 3 to the respondents' responses, respectively. The total cumulative score ranged from 0 to 24. A score of $0-5$ was considered as minimal anxiety, $6-10$ was considered mild anxiety, $11-15$ was considered moderate anxiety, while 
the range 16-24 was considered severe anxiety. Depression levels were assessed using PHQ-9, a 4-point Likert scale. The score $0-4$ was considered as no depression, 5-9 was considered as mild depression, 10-14 was considered as moderate depression, 15-19 was considered as moderately severe depression, and 20-27 was considered as severe depression. A 6-point Likert scale was used to assess the level of wellbeing. The total score of the five responses ranged from 0 to 25 . A score of $<13$ was considered the worst possible perceived quality of life, a score of $13-18$ scores was considered a moderate perceived quality of life, while a score of $>19$ was considered the best possible perceived quality of life. The correlation coefficient was interpreted using the following criteria: $0-0.25=$ weak correlation, $>0.25-0.5=$ fair correlation, $>0.5-0.75=$ good correlation, and greater than $0.75=$ excellent correlation .

\section{Validity and reliability}

Four experts (two epidemiologists and two specialists in infectious disease) have checked the content validity of the questionnaire items used in this study. The questionnaire has been adapted from English language versions and translated into the Arabic language. It was back-translated into English again to ensure content consistency in translation. Finally, the Arabic questionnaire version was pre-tested for ease of understanding of the questions by distributing 30 copies among healthcare workers. The modifications were made based on the responses in the pre-test phase.

\section{Data collection}

To avoid face-to-face contact and thus in-person interviewing due to the ongoing COVID-19 outbreak, an electronic web-based self-reported questionnaire was used to collect data, as recommended by the Ministry of Health and Population. The URL link was distributed to the HCWs via emails and social media including WhatsApp and Telegram. All HCWs who were working in the Yemeni health institutions, males and females, who were 18 years or older, and who agreed to participate were recruited for this study. The participants were encouraged to send the link to other colleagues as possible to increase the response rate of the survey. A total of $1,248 \mathrm{HCW}$ responded to the survey and are included in this study.

\section{Ethical consideration}

The study obtained ethical approval from Al-Razi University's Ethics Committee, and all methods were performed within the frame of the relevant guidelines and regulations. The objectives of the study were explained to the respondents. They were also informed that participation was voluntary and anonymity was assured and that they could withdraw from the study at any time. Before they could complete the web-based, self-report questionnaire, respondents had to confirm their voluntary participation by answering a yes-no question on the screen.

\section{Data analysis}


The collected data were managed and analyzed using Statistical Package for Social Sciences (IBM SPSS), version 24.0. Mean values and standard deviations were used to describe continuous variables, while dichotomous or categorical variables were described using the counts and percentages. Independent samples T-test and one-way ANOVA were used to compare the participants' demographic characteristics and levels of perceived stress, anxiety, insomnia, depression, and well-being. Spearman's correlation coefficients were used to find out the relation between stress, anxiety, insomnia, depression, and wellbeing scores. A p-value of $<0.05$ levels (two-tailed) with a 95 percent confidence interval was used to determine statistical significance.

\section{Results}

A total of 1,248 ( $72.8 \%$ males and $27.2 \%$ females) participated in this survey. The mean age of the participants was 32.5 years with 8.40 years SD. The majority were in the age group 18-30 years (47.2\%) and $31-40$ years (38.6\%). Approximately two-thirds of the sample $(67.0 \%)$ were married and $82.8 \%$ lived with family members. The occupations most frequently represented were respondents mainly physicians, nurses, and pharmacists $(31.5 \%, 21.1 \%$, and $11.0 \%$ respectively). About half of the participants $(50.2 \%)$ had achieved a bachelor's degree as their highest degree and the other educational levels were distributed as follows: Diploma (27.4\%), Master's (12.0\%), Medical Board (7.4\%), and Ph.D. (3.1\%). With regards to the respondents' working area, $17.5 \%$ worked in a non-specific unit, $11.9 \%$ in pharmacy, $9.2 \%$ in the emergency department, and $8.4 \%$ in the administration department. Most of the participants $(72.7 \%)$ had less than 10 years of working experience, and most worked 8 hours or less per day (67.3\%) and 4 to 6 days per week (80.3\%). More details are given in Table 1. The prevalence of moderate or severe stress (see Table 2) was $73.0 \%$ among the study participants, classified into moderate $(65.0 \%)$ and severe (8.0\%); only a quarter (27\%) reported low stress. Just under half of the respondents experienced symptoms of anxiety, with the overall prevalence of $49.8 \%$ distributed into these categories: mild (29.0\%), moderate $(14.7 \%)$, and severe (6.1\%). Insomnia was observed among $57.3 \%$ of the health staff which was categorized into mild (35.0\%), moderate (18.6\%), and severe (3.7\%). The prevalence of depression reported by the study participants was $54.3 \%$ of which severity varies as follows: mild (46.8\%), moderate $(7.4 \%)$, and moderately severe $(0.1 \%)$. Of the study subjects, $85.2 \%$ reported having the worst possible quality of life whereas $13.0 \%$ and $1.7 \%$ of them had a moderate and best possible quality of life respectively. Bivariate analysis of the associated factors of stress, anxiety, insomnia, depression, and wellbeing is shown in Table 3. Stress and insomnia scores were significantly higher among participants with $0-10$ years of experience ( $p$-value $0.002 \& 0.003$ respectively) and those working $>16$ hours per day (p-value $0.015 \& 0.036$ respectively). Moreover, anxiety score was also significantly higher among the subjects with $0-10$ years of experience, those working $>16$ hours per day and 7 days per week ( $p$-value $0.006,0.033 \& 0.030$ respectively). Divorced participants had higher depression scores than married, singles, and widows ( $p$-value 0.021 ) but this was not statistically significant. Wellbeing score showed that participants in the age groups; 31-40 years and 18-30 years had a significantly better quality of life than others ( $p$-value 0.027 ). Furthermore, a significantly better wellbeing score was reported among singles and married compared to divorced and widows ( $p$-value 0.018). There were significant positive 
correlations between stress and scores of anxiety, insomnia, and depression whereas a significant negative correlation was observed between stress and wellbeing scores. A significant positive correlation was also reported between anxiety and insomnia and depression and between insomnia and depression. Nevertheless, a significant negative correlation was between wellbeing scores and scores of anxiety, insomnia, and depression. Further details are provided in Table 4. 
Table 1

Demographic characteristics of the study participants

\begin{tabular}{|c|c|c|}
\hline \multicolumn{2}{|c|}{ Demographic characteristic } & \multirow{2}{*}{$\begin{array}{l}F(\%) \\
576(47.2)\end{array}$} \\
\hline Age & $18-30$ Years & \\
\hline & $31-40$ Years & $471(38.6)$ \\
\hline & $41-50$ Years & $120(9.8)$ \\
\hline & $51-60$ Years & $53(4.3)$ \\
\hline & Mean \pm SD & $32.53 \pm 8.40$ \\
\hline \multirow[t]{2}{*}{ Sex } & Male & $888(72.8)$ \\
\hline & Female & $332(27.2)$ \\
\hline \multirow[t]{4}{*}{ Marital Status } & Single & $385(31.6)$ \\
\hline & Married & $817(67.0)$ \\
\hline & Divorce & $5(0.4)$ \\
\hline & Widow & $13(1.1)$ \\
\hline \multirow[t]{2}{*}{ Living Status } & Living Alone & $210(17.2)$ \\
\hline & Living with Family & $1010(82.8)$ \\
\hline \multirow[t]{10}{*}{ Occupation } & Nurses & $257(21.1)$ \\
\hline & Physician & $384(31.5)$ \\
\hline & Anesthetist & $103(8.4)$ \\
\hline & Pharmacist & $134(11.0)$ \\
\hline & Administrative employee & $100(8.2)$ \\
\hline & Physiotherapist & $20(1.6)$ \\
\hline & Dentist & $47(3.9)$ \\
\hline & Laboratory personnel & $72(5.9)$ \\
\hline & Respiratory therapist & $83(6.8)$ \\
\hline & Radiologist & $20(1.6)$ \\
\hline \multirow[t]{4}{*}{ Educational Level } & Diploma & $334(27.4)$ \\
\hline & Bachelors & $612(50.2)$ \\
\hline & Master & $146(12.0)$ \\
\hline & Ph.D. & $38(3.1)$ \\
\hline
\end{tabular}




\begin{tabular}{|c|c|c|}
\hline \multicolumn{2}{|l|}{ Demographic characteristic } & \multirow{2}{*}{$\begin{array}{l}\mathbf{F}(\%) \\
90(7.4)\end{array}$} \\
\hline & Board & \\
\hline \multirow[t]{17}{*}{ Working Area } & Emergency Dep. & $112(9.2)$ \\
\hline & Anesthesia & $80(6.6)$ \\
\hline & Rehab/Physiotherapy center & $10(0.8)$ \\
\hline & Pharmacy & $145(11.9)$ \\
\hline & Pediatric Dep. & $35(2.9)$ \\
\hline & Internal Medicine Dep. & $76(6.2)$ \\
\hline & Surgical Dep. & $72(5.9)$ \\
\hline & Intensive Care Unit & $81(6.6)$ \\
\hline & Administration & $103(8.4)$ \\
\hline & Psychiatric and Mental Dep. & $29(2.4)$ \\
\hline & Orthopedic Dep. & $23(1.9)$ \\
\hline & Respiratory Therapy Dep. & $62(5.1)$ \\
\hline & Obstetrics \& Gynecology & $42(3.4)$ \\
\hline & Dentist clinics & $47(3.9)$ \\
\hline & Laboratory & $69(5.7)$ \\
\hline & Radiology Dep. & $20(1.6)$ \\
\hline & No specific unit & $214(17.5)$ \\
\hline \multirow[t]{4}{*}{ Experience Years } & $0-10$ Years & $887(72.7)$ \\
\hline & $10-20$ Years & $260(21.3)$ \\
\hline & $>20$ Years & $73(6.0)$ \\
\hline & Mean \pm SD & $7.75 \pm 6.81$ \\
\hline \multirow[t]{4}{*}{ Working Hours per day } & $0-8$ Hours & $821(67.3)$ \\
\hline & $9-16$ Hours & $373(30.6)$ \\
\hline & $>16$ Hours & $26(2.1)$ \\
\hline & Mean \pm SD & $8.97 \pm 3.38$ \\
\hline \multirow[t]{2}{*}{ Days of working per week } & 1-3 Days & $136(11.1)$ \\
\hline & 4-6 Days & $980(80.3)$ \\
\hline
\end{tabular}




\begin{tabular}{|lll|}
\hline Demographic characteristic & $\mathbf{F}(\%)$ \\
\hline 7 Days & $104(8.5)$ \\
\cline { 2 - 3 } Mean \pm SD & $\mathbf{5 . 2 3} \pm \mathbf{1 . 2 1}$ \\
\hline
\end{tabular}

Table 2

Prevalence of stress, anxiety, insomnia, depression and wellbeing quality

\begin{tabular}{|lll|}
\hline Variables & & $\mathbf{F}(\%)$ \\
\hline Stress & Low stress & $329(27.0)$ \\
\cline { 2 - 3 } & Moderate stress & $793(65.0)$ \\
\cline { 2 - 3 } Anxiety & High stress & $98(8.0)$ \\
\cline { 2 - 3 } & Minimal anxiety & $613(50.2)$ \\
\hline \multirow{5}{*}{ Insomnia } & Mild anxiety & $354(29.0)$ \\
\cline { 2 - 3 } & Moderate anxiety & $179(14.7)$ \\
\cline { 2 - 3 } & Sever anxiety & $74(6.1)$ \\
\cline { 2 - 3 } & Mild insomnia & $521(42.7)$ \\
\cline { 2 - 3 } & Moderate insomnia & $427(35.0)$ \\
\cline { 2 - 3 } & Sever insomnia & $227(18.6)$ \\
\hline Depression & No depression & $45(3.7)$ \\
\cline { 2 - 3 } & Mild depression & $558(45.7)$ \\
\cline { 2 - 3 } & Moderate depression & $571(46.8)$ \\
\cline { 2 - 3 } & Moderately severe depression & $1(0.1)$ \\
\hline Wellbeing & Worst possible quality of life & $1040(85.2)$ \\
\cline { 2 - 3 } & Moderate quality of life & $159(13.0)$ \\
\cline { 2 - 3 } & Best possible quality of life & $21.7)$ \\
\cline { 2 - 3 } & & $90.4)$ \\
\hline
\end{tabular}


Table 3

Factors affecting Stress, Anxiety, Insomnia, Depression and Wellbeing

\begin{tabular}{|c|c|c|c|c|c|c|}
\hline \multirow[t]{2}{*}{ Variable } & & Stress & \multirow{2}{*}{$\begin{array}{l}\text { Anxiety } \\
\text { Mean } \pm \\
\text { SD }\end{array}$} & \multirow{2}{*}{$\begin{array}{l}\text { Insomnia } \\
\text { Mean } \pm \\
\text { SD }\end{array}$} & \multirow{2}{*}{$\begin{array}{l}\text { Depression } \\
\text { Mean } \pm \text { SD }\end{array}$} & \multirow{2}{*}{$\begin{array}{l}\text { Wellbeing } \\
\text { Mean } \pm \\
\text { SD }\end{array}$} \\
\hline & & $\begin{array}{l}\text { Mean } \\
\pm \text { SD }\end{array}$ & & & & \\
\hline \multirow[t]{5}{*}{ Age } & $18-30$ Year & $\begin{array}{l}18.09 \\
\pm 5.88\end{array}$ & $\begin{array}{l}6.79 \pm \\
4.88\end{array}$ & $\begin{array}{l}9.96 \pm \\
6.09\end{array}$ & $5.06 \pm 2.71$ & $\begin{array}{l}9.16 \pm \\
3.69\end{array}$ \\
\hline & $31-40$ Year & $\begin{array}{l}17.55 \\
\pm 6.00\end{array}$ & $\begin{array}{l}6.64 \pm \\
5.16\end{array}$ & $\begin{array}{l}9.38 \pm \\
6.74\end{array}$ & $5.13 \pm 2.85$ & $\begin{array}{l}9.63 \pm \\
3.87\end{array}$ \\
\hline & 41-50 Year & $\begin{array}{l}16.86 \\
\pm 6.09\end{array}$ & $\begin{array}{l}5.55 \pm \\
4.34\end{array}$ & $\begin{array}{l}8.52 \pm \\
5.62\end{array}$ & $4.67 \pm 2.82$ & $\begin{array}{l}8.86 \pm \\
3.56\end{array}$ \\
\hline & $51-60$ Year & $\begin{array}{l}17.90 \\
\pm 6.30\end{array}$ & $\begin{array}{l}6.79 \pm \\
5.61\end{array}$ & $\begin{array}{l}9.13 \pm \\
6.86\end{array}$ & $4.81 \pm 3.15$ & $\begin{array}{l}8.39 \pm \\
3.39\end{array}$ \\
\hline & $p$ value & 0.165 & 0.102 & 0.104 & 0.397 & 0.027 \\
\hline \multirow[t]{3}{*}{ Sex } & Male & $\begin{array}{l}17.82 \\
\pm 5.96\end{array}$ & $\begin{array}{l}6.67 \pm \\
5.09\end{array}$ & $\begin{array}{l}9.55 \pm \\
6.45\end{array}$ & $4.95 \pm 2.77$ & $\begin{array}{l}9.22 \pm \\
3.65\end{array}$ \\
\hline & Female & $\begin{array}{l}17.56 \\
\pm 6.00\end{array}$ & $\begin{array}{l}6.43 \pm \\
4.66\end{array}$ & $\begin{array}{l}9.59 \pm \\
6.06\end{array}$ & $5.28 \pm 2.85$ & $\begin{array}{l}9.44 \pm \\
3.99\end{array}$ \\
\hline & $p$ value & 0.490 & 0.456 & 0.905 & 0.062 & 0.351 \\
\hline \multirow[t]{5}{*}{$\begin{array}{l}\text { Marital } \\
\text { Status }\end{array}$} & Single & $\begin{array}{l}17.84 \\
\pm 5.92\end{array}$ & $\begin{array}{l}6.31 \pm \\
4.73\end{array}$ & $\begin{array}{l}9.48 \pm \\
6.13\end{array}$ & $4.92 \pm 2.73$ & $\begin{array}{l}9.51 \pm \\
3.89\end{array}$ \\
\hline & Married & $\begin{array}{l}17.67 \\
\pm 5.99\end{array}$ & $\begin{array}{l}6.73 \pm \\
5.05\end{array}$ & $\begin{array}{l}9.56 \pm \\
6.43\end{array}$ & $5.09 \pm 2.82$ & $\begin{array}{l}9.22 \pm \\
3.68\end{array}$ \\
\hline & Divorce & $\begin{array}{l}22.00 \\
\pm 2.82\end{array}$ & $\begin{array}{l}9.60 \pm \\
6.46\end{array}$ & $\begin{array}{l}16.60 \pm \\
5.68\end{array}$ & $8.40 \pm 1.94$ & $\begin{array}{l}6.00 \pm \\
3.00\end{array}$ \\
\hline & Widow & $\begin{array}{l}18.38 \\
\pm 6.85\end{array}$ & $\begin{array}{l}7.07 \pm \\
6.76\end{array}$ & $\begin{array}{l}9.30 \pm \\
6.43\end{array}$ & $4.07 \pm 2.62$ & $\begin{array}{l}7.07 \pm \\
2.87\end{array}$ \\
\hline & $p$ value & 0.410 & 0.287 & 0.101 & 0.021 & 0.018 \\
\hline \multirow[t]{3}{*}{$\begin{array}{l}\text { Living } \\
\text { Status }\end{array}$} & Alone & $\begin{array}{l}17.92 \\
\pm 5.78\end{array}$ & $\begin{array}{l}6.92 \pm \\
5.20\end{array}$ & $\begin{array}{l}9.74 \pm \\
6.29\end{array}$ & $4.88 \pm 2.77$ & $\begin{array}{l}9.30 \pm \\
3.46\end{array}$ \\
\hline & with Family & $\begin{array}{l}17.72 \\
\pm 6.01\end{array}$ & $\begin{array}{l}6.54 \pm \\
4.93\end{array}$ & $\begin{array}{l}9.52 \pm \\
6.36\end{array}$ & $5.07 \pm 2.80$ & $\begin{array}{l}9.27 \pm \\
3.81\end{array}$ \\
\hline & $p$ value & 0.647 & 0.315 & 0.645 & 0.371 & 0.939 \\
\hline \multirow[t]{3}{*}{ Occupation } & Nurses & $\begin{array}{l}18.15 \\
\pm 6.26\end{array}$ & $\begin{array}{l}6.89 \pm \\
5.39\end{array}$ & $\begin{array}{l}9.82 \pm \\
6.58\end{array}$ & $5.04 \pm 2.94$ & $\begin{array}{l}9.23 \pm \\
4.40\end{array}$ \\
\hline & Physician & $\begin{array}{l}17.47 \\
\pm 6.00\end{array}$ & $\begin{array}{l}6.17 \pm \\
4.82\end{array}$ & $\begin{array}{l}9.16 \pm \\
6.14\end{array}$ & $4.84 \pm 2.61$ & $\begin{array}{l}9.29 \pm \\
3.38\end{array}$ \\
\hline & Anesthetist & 18.48 & $7.20 \pm$ & $9.65 \pm$ & $5.39 \pm 2.85$ & $9.36 \pm$ \\
\hline
\end{tabular}




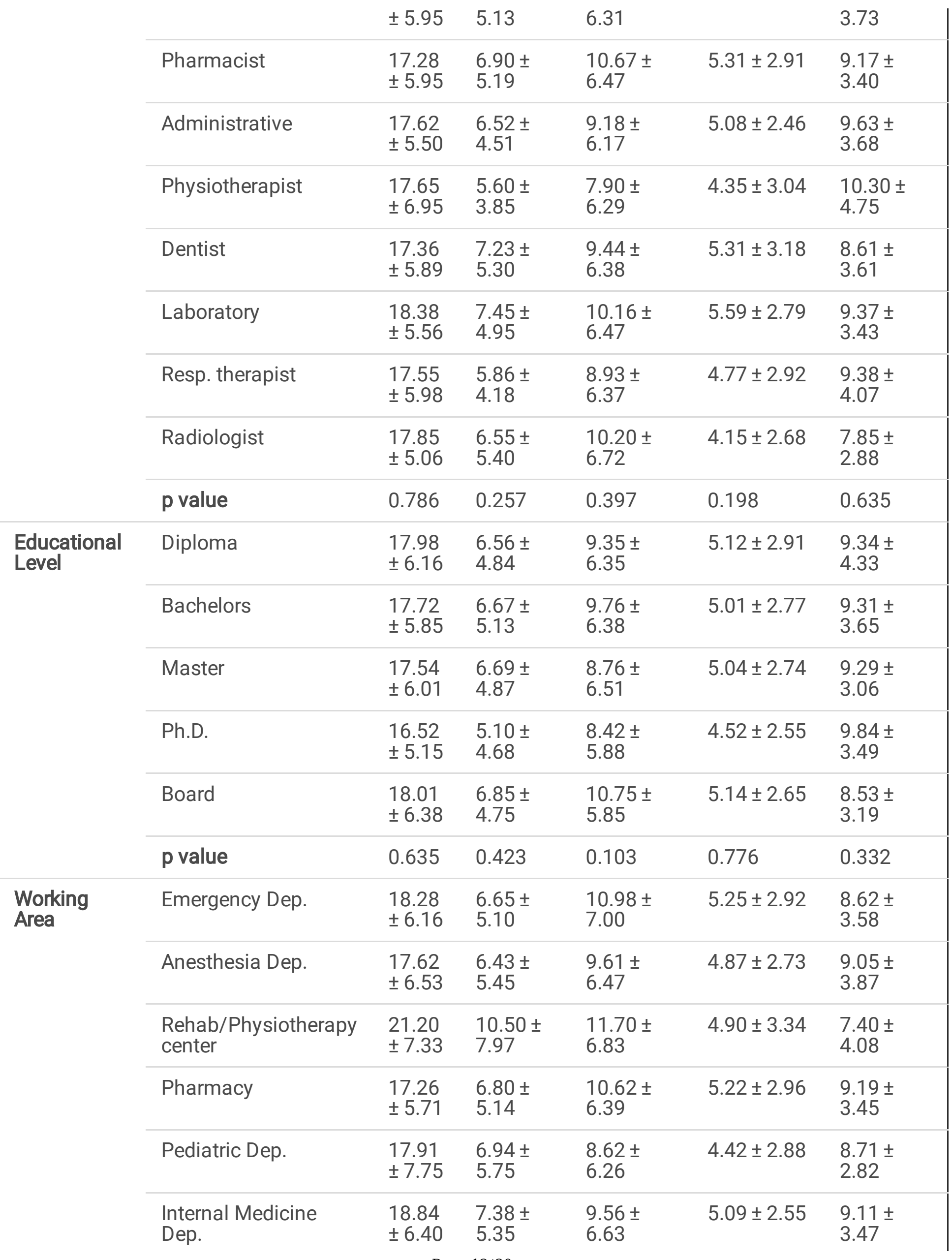




\begin{tabular}{|c|c|c|c|c|c|c|}
\hline & Surgical Dep. & $\begin{array}{l}17.34 \\
\pm 6.58\end{array}$ & $\begin{array}{l}5.97 \pm \\
4.98\end{array}$ & $\begin{array}{l}8.90 \pm \\
5.79\end{array}$ & $4.52 \pm 2.56$ & $\begin{array}{l}9.48 \pm \\
4.11\end{array}$ \\
\hline & Intensive Care Unit & $\begin{array}{l}17.43 \\
\pm 6.02\end{array}$ & $\begin{array}{l}6.16 \pm \\
4.7\end{array}$ & $\begin{array}{l}8.28 \pm \\
5.89\end{array}$ & $4.98 \pm 3.06$ & $\begin{array}{l}9.80 \pm \\
4.08\end{array}$ \\
\hline & Administration & $\begin{array}{l}17.64 \\
\pm 5.40\end{array}$ & $\begin{array}{l}6.51 \pm \\
4.56\end{array}$ & $\begin{array}{l}9.29 \pm \\
6.26\end{array}$ & $5.06 \pm 2.43$ & $\begin{array}{l}9.59 \pm \\
3.69\end{array}$ \\
\hline & $\begin{array}{l}\text { Psychiatric and } \\
\text { Mental }\end{array}$ & $\begin{array}{l}16.79 \\
\pm 6.23\end{array}$ & $\begin{array}{l}5.72 \pm \\
4.05\end{array}$ & $\begin{array}{l}8.62 \pm \\
5.51\end{array}$ & $4.55 \pm 2.54$ & $\begin{array}{l}10.24 \pm \\
3.90\end{array}$ \\
\hline & Orthopedic Dep. & $\begin{array}{l}17.65 \\
\pm 5.55\end{array}$ & $\begin{array}{l}6.82 \pm \\
5.08\end{array}$ & $\begin{array}{l}9.13 \pm \\
6.62\end{array}$ & $5.65 \pm 2.83$ & $\begin{array}{l}8.82 \pm \\
3.96\end{array}$ \\
\hline & $\begin{array}{l}\text { Respiratory Therapy } \\
\text { Dep. }\end{array}$ & $\begin{array}{l}17.93 \\
\pm 6.08\end{array}$ & $\begin{array}{l}6.40 \pm \\
4.82\end{array}$ & $\begin{array}{l}8.62 \pm \\
7.06\end{array}$ & $5.03 \pm 2.99$ & $\begin{array}{l}8.90 \pm \\
3.90\end{array}$ \\
\hline & $\begin{array}{l}\text { Obstetrics \& } \\
\text { Gynecology }\end{array}$ & $\begin{array}{l}16.85 \\
\pm 5.95\end{array}$ & $\begin{array}{l}4.85 \pm \\
3.52\end{array}$ & $\begin{array}{l}8.07 \pm \\
5.22\end{array}$ & $4.57 \pm 2.70$ & $\begin{array}{l}10.40 \pm \\
3.89\end{array}$ \\
\hline & No specific unit & $\begin{array}{l}17.84 \\
\pm 5.59\end{array}$ & $\begin{array}{l}6.57 \pm \\
4.74\end{array}$ & $\begin{array}{l}9.51 \pm \\
6.12\end{array}$ & $5.07 \pm 2.72$ & $\begin{array}{l}9.59 \pm \\
4.02\end{array}$ \\
\hline & Dentist clinics & $\begin{array}{l}17.36 \\
\pm 5.89\end{array}$ & $\begin{array}{l}7.23 \pm \\
5.30\end{array}$ & $\begin{array}{l}9.44 \pm \\
6.38\end{array}$ & $5.31 \pm 3.18$ & $\begin{array}{l}8.61 \pm \\
3.61\end{array}$ \\
\hline & Laboratory & $\begin{array}{l}18.08 \\
\pm 5.48\end{array}$ & $\begin{array}{l}7.42 \pm \\
4.90\end{array}$ & $\begin{array}{l}10.07 \pm \\
6.32\end{array}$ & $5.62 \pm 2.78$ & $\begin{array}{l}9.56 \pm \\
3.29\end{array}$ \\
\hline & Radiology Dep. & $\begin{array}{l}17.85 \\
\pm 5.06\end{array}$ & $\begin{array}{l}6.55 \pm \\
5.40\end{array}$ & $\begin{array}{l}10.20 \pm \\
6.72\end{array}$ & $4.15 \pm 2.68$ & $\begin{array}{l}7.85 \pm \\
2.88\end{array}$ \\
\hline & $p$ value & 0.836 & 0.263 & 0.184 & 0.569 & 0.123 \\
\hline \multirow[t]{4}{*}{$\begin{array}{l}\text { Experience } \\
\text { Years }\end{array}$} & $0-10$ Years & $\begin{array}{l}18.12 \\
\pm 5.87\end{array}$ & $\begin{array}{l}6.88 \pm \\
5.06\end{array}$ & $\begin{array}{l}9.91 \pm \\
6.31\end{array}$ & $5.14 \pm 2.77$ & $\begin{array}{l}9.33 \pm \\
3.68\end{array}$ \\
\hline & $10-20$ Years & $\begin{array}{l}16.88 \\
\pm 6.11\end{array}$ & $\begin{array}{l}5.98 \pm \\
4.76\end{array}$ & $\begin{array}{l}8.86 \pm \\
6.34\end{array}$ & $4.82 \pm 2.77$ & $\begin{array}{l}9.32 \pm \\
4.06\end{array}$ \\
\hline & $>20$ Years & $\begin{array}{l}16.38 \\
\pm 6.27\end{array}$ & $\begin{array}{l}5.53 \pm \\
4.49\end{array}$ & $\begin{array}{l}7.82 \pm \\
6.36\end{array}$ & $4.54 \pm 3.05$ & $\begin{array}{l}8.50 \pm \\
3.42\end{array}$ \\
\hline & p value & 0.002 & 0.006 & 0.003 & 0.081 & 0.191 \\
\hline \multirow{4}{*}{$\begin{array}{l}\text { Working } \\
\text { Hours per } \\
\text { day }\end{array}$} & $0-8$ Hours & $\begin{array}{l}17.44 \\
\pm 5.86\end{array}$ & $\begin{array}{l}6.43 \pm \\
4.90\end{array}$ & $\begin{array}{l}9.23 \pm \\
6.35\end{array}$ & $4.97 \pm 2.81$ & $\begin{array}{l}9.33 \pm \\
3.71\end{array}$ \\
\hline & $9-16$ Hours & $\begin{array}{l}18.29 \\
\pm 6.13\end{array}$ & $\begin{array}{l}6.85 \pm \\
5.07\end{array}$ & $\begin{array}{l}10.22 \pm \\
6.25\end{array}$ & $5.17 \pm 2.70$ & $\begin{array}{l}9.17 \pm \\
3.78\end{array}$ \\
\hline & $>16$ Hours & $\begin{array}{l}19.84 \\
\pm 6.48\end{array}$ & $\begin{array}{l}8.76 \pm \\
5.54\end{array}$ & $\begin{array}{l}10.42 \pm \\
6.88\end{array}$ & $5.07 \pm 3.58$ & $\begin{array}{l}9.03 \pm \\
4.47\end{array}$ \\
\hline & $\mathrm{p}$ value & 0.015 & 0.033 & 0.036 & 0.513 & 0.757 \\
\hline Working & 1-3 Days & 17.64 & $6.88 \pm$ & $9.01 \pm$ & $5.11 \pm 2.69$ & $9.76 \pm$ \\
\hline
\end{tabular}




\begin{tabular}{|c|c|c|c|c|c|c|}
\hline \multirow{4}{*}{$\begin{array}{l}\text { Days per } \\
\text { week }\end{array}$} & & \pm 5.44 & 5.04 & 6.27 & & 4.11 \\
\hline & 4-6 Days & $\begin{array}{r}17.78 \\
\pm 6.01\end{array}$ & $\begin{array}{l}6.45 \pm \\
4.89\end{array}$ & $\begin{array}{l}9.53 \pm \\
6.35\end{array}$ & $4.99 \pm 2.80$ & $\begin{array}{l}9.17 \pm \\
3.70\end{array}$ \\
\hline & 7 Days & $\begin{array}{l}17.58 \\
\pm 6.27\end{array}$ & $\begin{array}{l}7.76 \pm \\
5.57\end{array}$ & $\begin{array}{l}10.53 \pm \\
6.40\end{array}$ & $5.37 \pm 2.88$ & $\begin{array}{l}9.62 \pm \\
3.65\end{array}$ \\
\hline & $p$ value & 0.923 & 0.030 & 0.175 & 0.399 & 0.145 \\
\hline
\end{tabular}

Table 4

Correlation between Stress, Anxiety, Insomnia, Depression and Wellbeing

\begin{tabular}{|c|c|c|}
\hline Variable & Correlation coefficient & $P$-value \\
\hline Stress + Anxiety & $0.628^{* *}$ & $<0.001$ \\
\hline Stress + Insomnia & $0.493^{\star \star}$ & $<0.001$ \\
\hline Stress + Depression & $0.453^{\star \star}$ & $<0.001$ \\
\hline Stress + Wellbeing & $-0.095^{\star \star}$ & 0.001 \\
\hline Anxiety + Insomnia & $0.576^{\star *}$ & $<0.001$ \\
\hline Anxiety + Depression & $0.546^{\star \star}$ & $<0.001$ \\
\hline Anxiety + Wellbeing & $-0.204^{\star \star}$ & $<0.001$ \\
\hline Insomnia + Depression & $0.476^{\star *}$ & $<0.001$ \\
\hline Insomnia + Wellbeing & $-0.172^{\star *}$ & $<0.001$ \\
\hline Depression + Wellbeing & $-0.180^{\star \star}$ & $<0.001$ \\
\hline **. Correlation is signifi & at the 0.01 level (2-t & ed). \\
\hline
\end{tabular}

\section{Discussion}

The findings generated from this study paints a depressing picture of life as a healthcare worker in Yemen. Nearly three-quarters (73\%) of Yemeni HCWs report experiencing moderate or severe stress; nearly half $(49.8 \%)$ suffer from moderate or severe anxiety, a higher percentage suffer from insomnia (57.3\%), and a similar proportion from mild-severe depression (54.3\%). More than four-fifths (82.5\%) rated their quality of life to be in the worst possible category. The situation from which these conditions emerge is unlikely to improve shortly. Challenges with burnout amongst Yemeni HCWs have been recorded for more than a decade [22], as have challenges to establishing and supporting mental health programs in Yemen [23], leading to claims that the right to mental health care in the country is being ignored [24]. The findings of the current study are consistent with the results of previous studies [25-28] showing a 
considerable level of depression, anxiety, and insomnia among all HCWs during the COVID-19 outbreak. The results are also in line with the result of De Kock et al. [28], who found that the COVID-19 has a significant impact on the overall psychological wellbeing of front-line HCWs. Furthermore, the results are also in line with the results of Cai, H. et al. [29] and Alrubaiee et al. [17] regarding the high level of respondents' anxiety during the battle against the COVID-19 outbreak. In contrast, the result of this study seems to differ from the results of Huang and Zhao [30] and Zhang et al. [31], who found that HCWs have lower levels of anxiety, depression, and insomnia. These contradictory findings could be due to the differences in settings of the studies, availability of PPE, and the diversity of the respondents who participated in the study. It is not surprising, in this context, that many HCWs do not stay in Yemen for long once they qualify [32]. Of the total 1,248 respondents, $72.7 \%$ had less than 10 years of working experience, and $85.8 \%$ were under 40 years of age. This is indicative of the challenges Yemen faces with retaining HCWs who train in the country: many leave soon after qualification and this situation is unlikely to improve with the increased challenges the pandemic brings. Those who have remained in Yemen bear the scars of this. Statistically higher scores for stress $(p=0.02)$, anxiety $(p=0.06)$, and insomnia $(p=$ 0.03 ) were reported from those with more than 10 years of experience than those with less experience, and the older participants were also statistically significantly $(p=0.027)$ more likely to report the worst quality of life. This suggests that the older HCWs may not be staying in the highly stressful conditions they endure in Yemen by choice, or at the very least that they are far from happy with their current situation. These findings support the results reported by Alnazly et al. [28] that respondents' perceived depression, anxiety, and insomnia were significantly associated with the greater years of clinical experience. In contrast, the result seems to differ from the results of Alhurishi et al. [27], who stated that depression, anxiety, and insomnia among the respondents during the COVID-19 did not significantly differ based on the number of experience years. Regarding the perceived well-being of the participants, it was found to vary based on the respondents' age significantly. This corroborates with previous studies [30, 33], which reported a significant association between respondents' mental health such as generalized anxiety disorder, depressive symptoms, and their age. The exodus of Yemeni medics to safer positions and better-provisioned healthcare sectors elsewhere leaves those who remain likely to ensure long working hours, creating a vicious cycle of stress risk. Two-thirds of all HCWs (67.3\%) report working more than eight hours a day, and $8.5 \%$ work seven days a week. Those working more than 16 hours a day reported - hardly surprisingly - statistically significant higher levels of stress $(p=0.015)$, anxiety $(p=$ $0.033)$, and insomnia $(p=0.036)$. Those working more than 7 days a week were also more likely to report problems with anxiety $(p=0.030)$. This becomes even more concerning when one considers that those under 40 are statistically more likely $(p=0.028)$ to be the ones working longer hours than those in the older age groups. The increased risk of stress, anxiety, and insomnia associated with working longer hours is hardly likely to increase young medics' enthusiasm for remaining in Yemen (See supplementary table). This result is in line with a previous study [34] carried out in the United States, which indicated that longer working hours during the COVID-19 outbreak were highly associated with poorer psychological outcomes. The family situation provided interesting results that would require more research to fully understand. Divorced and widowed HCWs reported statistically higher scores for depression $(p=0.021)$ and lower scores for perceived wellbeing $(p=0.018)$ compared with those who were single but this may 
not be indicative of the conflict and humanitarian aid situation; it may simply be a reflection of the loss of, or separation from, a loved one that would be reflected within Yemeni society more widely. Further research would be needed to unpack this, particularly as there were no significant differences between respondents living alone and those living with family members. This is interesting as it may suggest that the fear of taking infection home to family members is being offset by the emotional support and companionship family members provide. Further qualitative work to understand the influence of these factors would add value to the understanding. There were no statistically significant differences observed between levels of reported stress, anxiety, depression, insomnia, or wellbeing based on gender, occupation within the hospital, level of qualification, or which hospital department respondents were employed in, suggesting that negative experiences are spread evenly but widely throughout the Yemeni healthcare workforce. Nevertheless, this study has several limitations which should be addressed for future research. First, our study was an electronic-based cross-sectional survey, hence we could not define the causal inferences; further qualitative work would be valuable to help understand specific drivers of stress. Second, we used an electronic web-based survey and voluntary sampling to avoid the potential transmission of COVID-19 infection; therefore, selection bias is possible, particularly as not all HCWs may have access to the internet. Third, the results of the self-reported survey should be taken with caution, as these results may differ from those obtained from face-to-face interviews; the conditions surveyed were not subject to clinical diagnosis.

\section{Conclusion}

Yemeni HCWs face unprecedented challenges, from internal conflict and civil strife, and now from COVID19. Considerable levels of stress, anxiety, depression, insomnia, and poor quality of life reported by HCWs shows an increasingly positive correlation with age, years of experience, and also with several hours and days worked; the latter is concentrated in the younger age groups, thus adding to their stress. These factors will increase the challenges Yemeni already faces in retaining its healthcare workforce for the future and presents a requirement on the healthcare sector to train more medical staff than it needs, as many are likely to leave for jobs elsewhere. This exodus puts an additional burden on an already underresourced and over-stretched system. In the short term, support for the Yemeni healthcare sector will be required from international aid agencies, who may need to be mindful of stationing their staff in-country for anything other than short periods to avoid exposing them to the risk factors experienced by the local staff. Psychological support to build resilience to the prevailing conditions may need to be embedded into medical school training and continuing professional development to help retain HCWs within Yemen.

\section{Abbreviations}

COVID-19: Coronavirus Disease 2019; SARS-CoV-2: Severe acute respiratory syndrome coronavirus2; HCWs: Healthcare workers; WHO: World Health Organisation; IBM SPSS: Statistical Package for Social Sciences; ICTV: International Committee on Taxonomy of Viruses; PPE: Personal protective equipment. 


\section{Declarations}

\section{Acknowledgements}

The authors would like to thank all the respondents for their participation and support in sharing the link to the questionnaire with other colleagues to participate.

\section{Authors' contributions}

MA and GGA were involved in the inception of the idea and study design. MA, GGA, TAHA and YA were responsible for the data collection. GGA, MA, YA and JC contributed to the literature review. FA and TAHA have performed the data analysis and created the tables. JC was contributed to the writing of the first draft of the discussion, suggested improvements and reviewed the manuscript. GGA, MA and JC have contributed to the writing of the final draft of the discussion, the interpretation of the findings and the review of the whole manuscript. MA participated and supervised the elaboration and every step of the paper writing process. All the authors contributed to drafting the manuscript and approving the final manuscript.

\section{Funding}

None.

\section{Availability of data and materials}

Data are available from the corresponding author on a reasonable request.

\section{Competing interests}

The authors declare that no competing interests.

\section{Ethics approval and consent to participate}

The Ethics Committee for Research of Al-Razi University approved this study prior to its conduct. Before the participants were allowed to participate, their consent was obtained to voluntarily participate by answering a "Yes or No" question in the online written informed consent form.

\section{Consent for publication}

Not applicable.

\section{References}

1. Wang C, Horby PW, Hayden FG, Gao GF: A novel coronavirus outbreak of global health concern. The lancet 2020, 395(10223):470-473. 
2. Organization WH: WHO Director-General's opening remarks at the media briefing on COVID-19-11 March 2020. In.: Geneva, Switzerland; 2020.

3. Huynh G, Nguyen TNH, Vo KN, Pham LA: Knowledge and attitude toward COVID-19 among healthcare workers at District 2 Hospital, Ho Chi Minh City. Asian Pacific Journal of Tropical Medicine 2020, 13(6):260.

4. Organization WH: Coronavirus COVID-19 Dashboard (https:// covid19. who. int/, 2020). In.: Accessed; 2020.

5. WHO: Coronavirus disease 2019 (COVID-19). Situation report-81. 2020. In.; 2020.

6. Ghebreyesus DTA: Director-General. World Health Organization (media briefing) 2020.

7. Ryu S, Chun BC, of Epidemiology KS: An interim review of the epidemiological characteristics of 2019 novel coronavirus. Epidemiology and health 2020, 42.

8. Wang D, Hu B, Hu C, Zhu F, Liu X, Zhang J, Wang B, Xiang H, Cheng Z, Xiong Y: Clinical characteristics of 138 hospitalized patients with 2019 novel coronavirus-infected pneumonia in Wuhan, China. Jama 2020, 323(11):1061-1069.

9. Necho M, Birkie M, Gelaye H, Beyene A, Belete A, Tsehay M: Depression, anxiety symptoms, Insomnia, and coping during the COVID-19 pandemic period among individuals living with disabilities in Ethiopia, 2020. PloS one 2020, 15(12):e0244530.

10. Brooks SK, Webster RK, Smith LE, Woodland L, Wessely S, Greenberg N, Rubin GJ: The psychological impact of quarantine and how to reduce it: rapid review of the evidence. The lancet 2020, 395(10227):912-920.

11. Chen J, Liu X, Wang D, Jin Y, He M, Ma Y, Zhao X, Song S, Zhang L, Xiang X: Risk factors for depression and anxiety in healthcare workers deployed during the COVID-19 outbreak in China. Social psychiatry and psychiatric epidemiology 2021, 56(1):47-55.

12. Li Y, Scherer N, Felix L, Kuper H: Prevalence of depression, anxiety and post-traumatic stress disorder in health care workers during the COVID-19 pandemic: A systematic review and meta-analysis. PloS one 2021, 16(3):e0246454.

13. MedGlobal: A tipping point for Yemen's health system: the impact of COVID-19 in a fragile state. 2020.

14. Al-Awlaqi S, Dureab F, Annuzaili D, Al-Dheeb N: COVID-19 in Conflict: The devastating impact of withdrawing humanitarian support on universal health coverage in Yemen. Public Health in Practice 2020, 1:100015.

15. Islam Z, Rocha ICN, Mohanan P, Jain S, Goyal S, dos Santos Costa AC, Ahmad S, Mehedi Hasan M, Essar MY: Mental health impacts of humanitarian crisis on healthcare workers in Yemen. Medicine, Conflict and Survival 2021:1-6.

16. Elnakib S, Elaraby S, Othman F, BaSaleem H, AlShawafi NAA, Al-Gawfi IAS, Shafique F, Al-Kubati E, Rafique N, Tappis $\mathrm{H}$ : Providing care under extreme adversity: The impact of the Yemen conflict on the personal and professional lives of health workers. Social Science \& Medicine 2021, 272:113751. 
17. Alrubaiee GG, Al-Qalah TAH, Al-Aawar MSA: Knowledge, attitudes, anxiety, and preventive behaviours towards COVID-19 among health care providers in Yemen: an online cross-sectional survey. BMC Public Health 2020, 20(1):1-11.

18. Noushad M, Al-Saqqaf IS: COVID-19 case fatality rates can be highly misleading in resource-poor and fragile nations: the case of Yemen. Clinical Microbiology and Infection 2021, 27(4):509-510.

19. Organization WH: COVID-19: Occupational health and safety for health workers: interim guidance, 2 February 2021. In.: World Health Organization; 2021.

20. Papandrea D, Azzi M: Managing work-related psychosocial risks during the COVID-19 pandemic. International Labour Organization 2020.

21. Badahdah AM, Khamis F, Al Mahyijari N: The psychological well-being of physicians during COVID19 outbreak in Oman. Psychiatry research 2020, 289:113053.

22. Al-Dubai SAR, Rampal KG: Prevalence and associated factors of burnout among doctors in Yemen. Journal of occupational health 2009:0911040104-0911040104.

23. Saleh MABQ, Makki AM: Mental health in Yemen: Obstacles and challenges. International Psychiatry 2008, 5(4):90-92.

24. ALHARIRI W, MCNALLY A, KNUCKEY S: The Right to Mental Health in Yemen: A Distressed and Ignored Foundation for Peace. 2021.

25. Ahn MH, Shin Y-W, Suh S, Kim JH, Kim HJ, Lee K-U, Chung S: High work-related stress and anxiety response to COVID-19 among healthcare workers in South Korea: SAVE study. 2020.

26. Asnakew S, Amha H, Kassew T: Mental Health Adverse Effects of COVID-19 Pandemic on Health Care Workers in North West Ethiopia: A Multicenter Cross-Sectional Study. Neuropsychiatric Disease and Treatment 2021, 17:1375.

27. Alhurishi SA, Almutairi KM, Vinluan JM, Aboshaiqah AE, Marie MA: Mental Health Outcomes of Healthcare Providers During COVID-19 Pandemic in Saudi Arabia: A Cross-Sectional Study. Frontiers in Public Health 2021, 9:647.

28. Alnazly E, Khraisat OM, Al-Bashaireh AM, Bryant CL: Anxiety, depression, stress, fear and social support during COVID-19 pandemic among Jordanian healthcare workers. Plos one 2021, 16(3): $\mathrm{e} 0247679$.

29. Cai H, Tu B, Ma J, Chen L, Fu L, Jiang Y, Zhuang Q: Psychological impact and coping strategies of frontline medical staff in Hunan between January and March 2020 during the outbreak of coronavirus disease 2019 (COVID-19) in Hubei, China. Medical science monitor: international medical journal of experimental and clinical research 2020, 26:e924171-924171.

30. Huang Y, Zhao N: Generalized anxiety disorder, depressive symptoms and sleep quality during COVID-19 outbreak in China: a web-based cross-sectional survey. Psychiatry research 2020, 288:112954.

31. Zhang W-r, Wang K, Yin L, Zhao W-f, Xue Q, Peng M, Min B-q, Tian Q, Leng H-x, Du J-I: Mental health and psychosocial problems of medical health workers during the COVID-19 epidemic in China. Psychotherapy and psychosomatics 2020, 89(4):242-250. 
32. Alsabri M, Nightingale B, Amin M, Cole J: When COVID-19 hit Yemen: dealing with the pandemic in a country under pressure from the world's worst humanitarian crisis.

33. Rossi R, Socci V, Pacitti F, Mensi S, Di Marco A, Siracusano A, Di Lorenzo G: Mental health outcomes among healthcare workers and the general population during the COVID-19 in Italy. Frontiers in Psychology 2020, 11.

34. Sagherian K, Steege LM, Cobb SJ, Cho H: Insomnia, fatigue and psychosocial well-being during COVID-19 pandemic: A cross-sectional survey of hospital nursing staff in the United States. Journal of clinical nursing 2020.

35. Supplementary table: Relationship of age of HCW to number of hours worked per day

\section{Supplementary Files}

This is a list of supplementary files associated with this preprint. Click to download.

- SupplementaryA.docx 